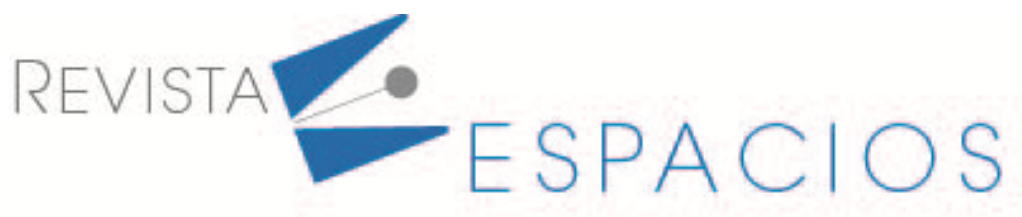

\title{
Lectura del avance del ODS 6 en América Latina y el Caribe
}

\section{Reading of the progress of SDG 6 in Latin America and the Caribbean}

\author{
URAZÁN BONELLS, Carlos Felipe ${ }^{1}$ \\ CAICEDO LONDOÑO, María Alejandra ${ }^{2}$ \\ AYALA ROJAS, Luis Efrén ${ }^{3}$ \\ LONDOÑO LINARES, Juan Pablo ${ }^{4}$
}

\begin{abstract}
Resumen
Este estudio analiza la correlación entre la condición económica de un país y su proyección para cumplir en 2030 las metas del ODS 6 (agua y saneamiento). La proyección se realizó con registros entre los años 2000 y 2017 . Se concluye que un país con buen nivel económico tiende a avanzar en la extensión de sus redes de servicios básicos, y esa relación es de mayor tendencia a la cobertura del servicio de saneamiento, que al de abastecimiento de agua.
\end{abstract}

Palabras clave: abastecimiento de agua segura, objetivos de desarrollo sostenible ods, saneamiento básico adecuado

\begin{abstract}
His study analyzes the correlation between the economic condition of a country and its projection to meet the goals of SDG 6 (water and sanitation) by 2030. The projection was made with records between the years 2000 and 2017. Its concluded that a country with a good economic level tends to advance in the extension of its basic service networks, and that relationship is more likely to cover the sanitation service, than the water supply.
\end{abstract}

key words: safe water supply, sustainable development goals sdg, adequate basic sanitation

\section{Introducción}

Hoy por hoy la guía del desarrollo mundial está enfocada en los Objetivos de Desarrollo Sostenible ODS (ONU, 2020 a). Es importante recordar que los ODS son una evolución de los Objetivos del Milenio ODM, que planteó la primera lista de metas globales que permitirían mejorar la calidad de vida de la población mundial con mira al año 2015 (las metas de cada país correspondían a condiciones que se proyectaban como factibles o posibles de alcanzar a esa fecha) (PNUD, 2020 a).

Como no todos los indicadores se habrían cumplido en el año 2015, en función de la aspiración de reducir significativamente pobreza, se dio continuidad al esfuerzo con el planteamiento de los 17 ODS, de los cuales el número 6 se denomina "Agua limpia y saneamiento", tema que venía trabajándose desde el ODM 7 "Garantizar

\footnotetext{
${ }^{1}$ Docente investigador del programa de Ingeniería Civil de la Universidad de La Salle, Bogotá, Colombia. Email: caurazan@unisalle.edu.co.

2 Docente investigador del programa de Ingeniería Civil de la Universidad de La Salle, Bogotá, Colombia. Email: macaicedo@unisalle.edu.co.

${ }^{3}$ Docente investigador del programa de Ingeniería Civil de la Universidad de La Salle, Bogotá, Colombia. Email: layalar@unisalle.edu.co.

4 Universidad Politécnica de Cataluña. Investigador del programa de Ingeniería Civil de la Universidad de La Salle, Bogotá, Colombia. Email: julondono@lasalle.edu.co.
} 
la sostenibilidad del medio ambiente" y la Meta 7C "Reducir a la mitad, para el año 2015, el porcentaje de personas que carezcan de acceso sostenible a agua potable".

El presente estudio aborda un análisis de la progresión de la cobertura de los servicios de abastecimiento de agua y saneamiento en la región latinoamericana y del Caribe, desde el año 2000, hasta los últimos registros oficiales del año 2019, vinculándolos con el incremento poblacional y económico (a partir del PIB per cápita). El objetivo principal fue determinar el período quinquenal, a partir de año 2000, en el cual los países seleccionados lograrían cubrir el $100 \%$ de su respectiva población con los servicios de agua segura para consumo, y acceso a servicios adecuados de saneamiento básico.

Este estudio se enmarca como parte del proyecto "Condiciones de infraestructura vial, abastecimiento de agua y saneamiento en zonas rurales colombianas, en procura de los Objetivos de Desarrollo Sostenible (ODS)" (2019), desarrollado por los presentes autores, como grupo de trabajo del Programa de Ingeniería Civil de la Universidad de La Salle. El proyecto es financiado por la Vicerrectoría de Investigaciones y Transferencia VRIT, de la misma universidad.

\subsection{Del ODM 7C al ODS 6}

Los Objetivos de Desarrollo del Milenio ODM, se enmarcan en el Fondo de los Objetivos de Desarrollo Sostenible (ONU, 2020 b). La denominada Conferencia Rio+20 (Conferencia de las Naciones Unidas sobre el desarrollo sostenible; www.rio20.net), llevada a cabo en el año 2012, fue la plataforma para dar paso a los Objetivos de Desarrollo Sostenible ODS.

El Informe del año 2015 sobre los ODM (ONU, 2015) expresa el logro específico al pasar de 2.300 millones a 4.300 de personas que a nivel mundial tuvieron acceso a agua potable suministrada por cañería, esto entre los años 1990 y 2015. A nivel regional, América Latina y el Caribe incrementó el porcentaje entre los años 1990 y 2015, pasando del $85 \%$ al $95 \%$. Con esa última cifra se sobrepasa la meta regional para el año 2015 . Esa meta no fue cumplida en su momento (2015) por Oceanía, el Cáucaso y Asia Central, y África septentrional.

También se hace mención del avance del acceso a servicios de saneamiento mejorado a nivel mundial, el cual aumentó en el mismo período (1990 a 2015) en 2.100 millones de personas; y del porcentaje de personas que defecan al aire libre que se ha reducido casi a la mitad desde 1990. A nivel regional (América Latina y el Caribe), la cobertura poblacional es más baja que con el tema agua, pues entre los años 1990 y 2015, se pasó del 67\% al 83\%, por lo cual tampoco cumplió la meta del 85\%. Ese indicador solo logró su meta a 2015 en Asia oriental, Asia occidental, África septentrional, y Cáucaso y Asia central.

Esa mejor condición de vida influyó, sin lugar a duda, en que el porcentaje de población urbana que vive en barrios marginales se redujo de cerca del $40 \%$ al 30\%, entre los años 2000 y 2014 . A nivel regional, América Latina y El Caribe mejoró la condición al pasar del 29\% al 20\%. Ese 20\% en 2014 posiciona a la región en mejor situación que África subsahariana, Asia meridional, Asia sudoriental, Asia oriental, Asia occidental, y Oceanía. Solo es superado por África septentrional. Se recuerda que la comparación que realiza el documento oficial es respecto de las regiones del mundo que se consideran como Regiones en desarrollo.

Urazán y Caicedo (2018) publicaron un estudio que compara las correlaciones entre los servicios públicos de agua y saneamiento, con la cantidad poblacional y la economía de países latinoamericanos y del Caribe, pero con ventanas específicas para los años 1990, 2000 y 2015; en función de los indicadores de los ODM. Las conclusiones establecen que con el paso de los años, la condición económica de los países estudiados (PIB) disminuyó su impacto o participación (medido por el valor de su coeficiente como variable explicativa de un modelo de correlación lineal múltiple) respecto de la cobertura en saneamiento básico; y en mayor margen lo ha hecho la población total nacional. 
En otro estudio similar (Urazán, Caicedo,2018), se concluye que el reto de los países latinoamericanos, con miras a los ODM, es lograr atener la demanda futura de servicios públicos básicos ante el creciente aumento de la población urbana en los distintos países de la región; ya que el fenómeno de la urbanización (migración campo a ciudad) no se detiene. Ese efecto migratorio suele acompañarse de generación de pobreza urbana, que se manifiesta en la generación de tugurios o asentamientos informales, complicando aún más la aplicación de estrategias para cobijar a más población con la prestación de los servicios básicos de agua y saneamiento; con la mejora material y estructural de las viviendas, y lograr llegar al punto de la legalización predial como fin último del proceso urbano.

\subsection{Avance y retos del ODS 6}

El reporte oficial del avance de los ODS (Organización de las Naciones Unidas, 2020 c) para 2020, establece a pesar de todos los esfuerzos realizados por ampliar las cifras de los indicadores del ODS 6, aún hay 2.200 millones de personas sin acceso a agua potable manejada de manera segura; y cerca de 4.200 millones carecen de servicios de saneamiento gestionados de manera adecuada (cifras a 2017, reportadas en el informe 2020).

Las cifras también mencionan que 3.000 millones de personas no cuentan con instalaciones básicas para para lavado de manos en el hogar (medida fundamental para avanzar frente al COVID-19). De otro lado, dos de cada cinco centros de salud no tienen acceso a jabón, agua y desinfectantes (2016).

Como impacto social directo, se estima que la escasez de agua podría desplazar a unos 700 millones de habitantes para el año 2030; y algunos países registraron un déficit del 61\% para cumplir las metas en materia de agua y saneamiento; y como dato ligado a la carencia de servicios básicos, se tiene que la población urbana que habita sectores marginales creció un $24 \%$ en el año 2018.

Otra fuente de la Organización de las Naciones Unidas (ONU, 2020 d) destaca que al menos 892 millones de personas continúan con la práctica de defecación a cielo abierto, y que 4 billones de personas no cuentan con servicios sanitarios como retretes o letrinas. Que en el $80 \%$ de los casos son mujeres y niños quienes deben recolectar agua fuera del hogar y que más del $80 \%$ de las aguas residuales de actividades humanas son vertidas a cuerpos naturales de agua sin recibir tratamiento alguno.

La misma fuente recuerda que entre las metas a nivel mundial para el ODS 6 en 2030 se encuentran: lograr acceso universal y equitativo al agua potable a un precio razonable; lograr acceso universal a servicios sanitarios e higiénicos adecuados, y eliminar los casos de defecación a cielo abierto; y asegurar la sostenibilidad de la extracción y abastecimiento de agua dulce con el fin de reducir la escasez.

Otro documento paralelo de la Organización de las Naciones Unidas (ONU, 2020 e) complementa las cifras anteriores y recuerda que cerca de 1.800 millones de personas en el mundo consumen agua contaminada con residuos de heces fecales; y que la falta de acceso al agua afecta hoy por hoy al $40 \%$ de la población mundial.

Por tanto, la contaminación derivada de la carencia de servicios adecuados en agua y saneamiento pone de manifiesto la relación con enfermedades derivadas, causa de que más de 800 niños mueran al año por causa de problemas diarreicos. Además, la adecuada gestión del agua permite una mejor gestión en la producción de alimentos y energía, por ende, deriva en apoyo al crecimiento económico de comunidades.

Ahora, la Organización Mundial de la Salud expone que en busca de cumplir los objetivos en materia de agua y saneamiento para el año 2030 se requiere una inversión cercana a los 114 billones U\$/año, cifra que no incluye costos de operación y mantenimiento (OMS, 2017).

Bajo la lente regional para América Latina y el Caribe, las cifras con cierre a 2017 establecen que: la cobertura poblacional de servicios de agua en condiciones básicas y gestionadas de manera adecuada suman el $96.8 \%$, 
mientras que a nivel global (mundial) la cifra es de $89.6 \%$ y en los países en vía de desarrollo es del $64.6 \%$. Pasando a los registros de saneamiento básico y gestionados de manera adecuada, el porcentaje latinoamericano es de $87.2 \%$, el global asciende a $73.5 \%$, y el de las regiones en desarrollo a $34.2 \%$. Las cifras ponen a América Latina y el Caribe como una región que ha demostrado un avance significativo, pero aún con saldos pendientes a la ventana del año 2030 (CEPAL, 2020 a).

\subsection{Correspondencia entre el ODS 6 y el ODS 11}

El análisis del Objetivo de Desarrollo Sostenible ODS 11, establece que más de la mitad de la población mundial habita hoy por hoy en ciudades, y que para el año 2050 la cantidad superará los 6.500 millones de habitantes urbanos, cerca de un $66 \%$ de la población mundial (PNUD, 2020 b). Las cifras también mencionan que cerca de 828 millones de personas habitan en barrios marginales urbanos, y la cifra seguirá aumentando. Ese crecimiento poblacional urbano está ligado a movimientos migratorios que se definen bajo condiciones de desigualdad social y económica.

En el caso latinoamericano el proceso de urbanización supera el 80\%, acompañado de generación de pobreza y desigualdad, expresado en la presencia de tugurios, que se define como "un grupo de personas que vive bajo el mismo techo en una zona urbana que carece de una o más de las siguientes condiciones: 1 . Una vivienda durable de naturaleza permanente que proteja contra condiciones climáticas adversas. 2. Un espacio vital suficiente, lo que significa que no más de tres personas compartan una habitación. 3. Acceso fácil a agua potable en cantidad suficiente y a un precio razonable. 4. Acceso a saneamiento adecuado: letrina privada o pública compartida con una cantidad razonable de personas. 5. Tenencia segura para evitar los desalojos forzados" (CEPAL, 2017). Como se aprecia, las condiciones 3 y 4 hacen referencia a condiciones de abastecimiento de agua y de saneamiento, en condiciones adecuadas. Por tanto, el cumplimiento de las metas del ODS 6 en sectores de periferia informal urbana, está ligado a un avance en el cumplimiento del ODS 11. Otros documentos (Urazán, 2008) dan cuenta de esa relación entre la extensión paulatina de las redes de acueducto y alcantarillado a sectores de periferia urbana y el avance urbanístico del paso de tugurios a sectores reglamentados o legalizados.

Otro estudio (Tezanos, 2019) analiza el comportamiento de 16 países latinoamericanos y del Caribe respecto de su comportamiento en términos de ingresos económicos con miras a los resultados esperados por los ODS en el año 2030. Concluye la existencia de 3 grupos de países: unos con baja pobreza pero con aumento lento en el ingreso de los pobres; otros con altos índices de pobreza y también bajos ritmos en el crecimiento del ingreso de las personas con menos recursos; y países con tasas medias para ambas variables.

Por su parte, en el caso colombiano, el documento del Consejo Nacional de Política Económica y Social CONPES 3918 de 2018, expone los resultados que el país obtuvo en los ODM, y su fundamento para el planteamiento con que se establecieron los ODS.

\subsection{Condición en agua potable y saneamiento en América Latina}

El Banco Interamericano de Desarrollo BID, publica en su página web expone casos de habitantes que hoy por hoy tienen el servicio de baño en letrinas comunitarias en las que se exponen riesgos salubres e incluso de seguridad personal. Así vive cerca del $69 \%$ de la población latinoamericana, unos 490 millones de personas.

Además, cerca de 19 millones de personas aún practican la defecación a cielo abierto, con sus consecuentes problemas de salud e impacto al medioambiente.

El servicio de saneamiento mejorado ha sido una tarea rezagada respecto de la cobertura de agua potable. Solo el 20 de las aguas residuales de la región (provenientes de aguas servidas de vivienda o de otra actividad) tiene 
tratamiento previo a ser vertidas en fuentes naturales. Esto complica más el impacto ambiental por falta de infraestructura adecuada para el saneamiento (BID, 2020).

En 2019 se llevó a cabo la semana mundial del agua (SIWI - World Water Week), en Estocolmo, Suecia. Como parte de la agenda, se tiene el apartado "Saneamiento óptimo: Foco en las Américas durante la Semana Mundial del Agua 2019" en el cual se resalta que solo el $22 \%$ de la población en la región cuenta con acceso al servicio mencionado; a la vez que sugiere cambiar el enfoque tradicional para cerrar las brechas de acceso a agua potable y servicios fiables de saneamiento. Por tanto, "se requiere hallar nuevos enfoques para el desarrollo y planificación en un escenario mundial con menos recursos financieros destinados a agua y saneamiento", menciona la web del evento (SIWI, 2019).

Castro y Valbuena (2020) expone el avance de los países de la región respecto de los ODS, a corte del año 2019. Entre los ODS analizados está el número 6, correspondiente a la temática que nos atañe en el presente documento. Como resultado los países con mejor desempeño a corte 2019 son: Chile, Costa Rica y Uruguay; y da un valor medio de calificación a la región de 63.1. Los mayores atrasos están por un lado en la zona Caribe en: Haití y Trinidad y Tobago; y en Centroamérica en: Guatemala, Guyana, Belice, Honduras y Venezuela. Respecto del ODS 6, tuvo cumplimiento del 100\% solo por Chile y Uruguay; con calificación de rezago crítico solo está Haití; con rezago significativo están: Bolivia, Guatemala, Nicaragua, Perú y República Dominicana. Los demás países de la región se clasifican con rezago moderado.

El estudio de Pinilla y Torres (2019), establece la relación entre le gasto público social y la cobertura de los servicios de agua y saneamiento entre los años 1994 y 2014, separando el análisis de población urbana del rural. La principal conclusión es la existencia de una relación constante y positiva entre las 2 variables en el escenario rural; situación en la cual el aporte estatal ha tenido que ser mayor por la dificultad de acceso a las redes.

La expectativa de que la región latinoamericana y del Caribe puede cumplir la cobertura total en los servicios poblacionales de agua potable y saneamiento adecuado, se plantea al menos desde el año 2012. En ese año el Banco de Desarrollo de América Latina CAF, manifestaba en el documento: Agua potable y saneamiento básico en América Latina y El Caribe: metas realistas y soluciones sostenibles (CAF, 2012) que "para 2030, América Latina puede cerrar la brecha de los servicios de agua potable y saneamiento básico, con inversiones anuales equivalentes al $0,3 \%$ del PIB y mejoras substanciales de la gobernabilidad sectorial". La lectura de los informes más recientes respecto al tema, dejan de manifiesto que la cobertura en saneamiento mejorado tiene por delante un trabajo pendiente importante.

Como estrategias para lograr las metas a 2030, autores como Paltán, Bizzani, Minaya y Rezzano (2020) exponen la importancia de plantear sistemas resilientes de abastecimiento de agua y saneamiento en la región, en función de criterios de: resiliencia (capacidad de un sistema para recuperarse y mantener un desempeño adecuado a pesar de afrontar situaciones complejas), flexibilidad (capacidad de un sistema para ajustarse a nuevas condiciones), robustez (capacidad de un sistema para mantener un desempeño adecuado ante diferentes situaciones), confiabilidad (posibilidad de que un sistema falle), y vulnerabilidad (estimación de lo crítico de una falla). No obstante, los autores manifiestan la complejidad de establecer sistemas de servicios que cumplan con esos condicionantes, pero que de lograrse sustentarían la provisión de los servicios a lo largo del tiempo.

\section{Metodología}

El presente estudio está fundamentado en el cruce de información oficial, obtenida a través de páginas web y documentos de entidades ligadas a la Organización de las Naciones Unidas. El que las fuentes provengan de una misma entidad y sus filiales, garantiza unificación en las fichas metodológicas de las distintas variables analizadas. 
Inicialmente se definieron las variables que podrían correlacionarse con el avance de los países latinoamericanos y del Caribe en el cumplimiento del ODS 6. Entre ellas están: el nivel económico del país (medido a través del PIB per cápita, a precios constantes), la cantidad poblacional nacional, y la proporción de habitantes urbanos.

Luego se definió una lista de países latinoamericanos y del Caribe.

Como el objetivo principal es determinar el período quinquenal, a partir de año 2000, en el cual los países seleccionados lograrían cubrir el $100 \%$ de su respectiva población con los servicios de agua segura para consumo, y acceso a servicios adecuados de saneamiento básico; se encontraron las cifras retroactivas de los indicadores analizados.

Con las series históricas se proyectó el período en el cual cada uno de los países contarían con cobertura del $100 \%$ poblacional, tanto a nivel urbano como rural. La proyección está basada en la ecuación 1:

$$
y=x *(1+t a)^{\wedge} n \quad \text { Ec. } 1
$$

Donde:

$$
\begin{aligned}
& y=\text { valor proyectado de la variable } \\
& x=\text { valor de la variable en el año base } \\
& \text { ta }=\text { tasa de crecimiento anual de la variable } \\
& n=\text { número de años a proyectar }
\end{aligned}
$$

Cabe recordar que se trabajó sobre series históricas de años en los que el comportamiento económico y social (crecimiento poblacional) conservaba una tendencia similar; a diferencia de los análisis que puedan incluir las actuales afectaciones a nivel mundial, debidas a la pandemia del COVID-19. Este análisis es válido si se considera que las externalidades negativas de la pandemia serán superadas en gran medida en el año 2021.

Posteriormente, se realizaron correlaciones lineales múltiples para determinar variables que explicaran, a nivel regional, un mayor avance en la cobertura de población que usa fuentes mejoradas de agua y de población que usa servicios sanitarios seguros.

Para las correlaciones se empleó la herramienta "análisis de datos" para "regresiones", en una hoja de cálculo (Excel). La validez estadística de las correlaciones planteadas se basó en un valor de $\mathrm{R}^{2}$ ajustado que fuese igual o superior a 0.75 . De obtenerse un valor de $R^{2}$ ajustado que se considere válido, se da revisión al signo de los coeficientes de correlación para las variables explicativas. Si los anteriores criterios coinciden con la hipótesis, se da validez a la correlación.

En los distintos planteamientos se emplearon 3 variables explicativas, luego se correlacionó en grupos combinados de 2 variables explicativas (de las 3 anteriores), y finalmente una correlación lineal de 1 variable explicativa (con cada una de las 3 iniciales). En conjunto para cada hipótesis planteada se realizaron 7 correlaciones (1 de 3 variables, 3 de 2 variables, y 3 de 1 variable) para comprobar su validez estadística.

\section{Resultados}

Inicialmente, se ha establecido el avance hasta los registros más recientes (2017), a partir del año 2000, de los 16 países seleccionados, respecto de: el porcentaje de la población que usa fuentes mejoradas de agua, y del porcentaje poblacional que usa servicios sanitarios seguros (figuras 1 a 5). Los países seleccionados fueron: 
En un escenario inicial (2000) (figura 1) se observa mayor cobertura poblacional para las fuentes mejoradas de agua (80\% para Bolivia como menor valor) respecto de la de saneamiento seguro (52\% como menor valor para Bolivia). Solo hay un grupo de 5 países con ambas coberturas por encima de 90\% (Uruguay, Costa Rica, República Dominicana, Chile y Argentina), y solo Uruguay registraba los 2 indicadores mayores a 95\%.

Para el año 2005 (figura 2), siguen siendo los mismos 5 países con registro por encima de 90\%, pero ahora son 3 de ellos (Uruguay, Costa Rica y Chile) con valores conjuntos que superan el $95 \%$ de población. No obstante, cabe aclarar que entre 2000 y 2005 hay avance para todos los países, en ambos servicios.

Luego, al comparar las cifras de 2005 y 2010 (figuras 2 y 3) se incrementó a 7 el número de países con indicadores mayores a 90\% (se unen Colombia y México); y 3 los que superan el 95\% (Uruguay, Costa Rica y Chile). Nuevamente, todos los países presentaron mejoras comparativas para los 2 servicios.

La comparación entre los registros de 2010 y 2015 (figuras 3 y 4) muestra cambios significativos. Al grupo de países con registros superiores al 90\% se suman Ecuador y Paraguay (9 países de 16); y el grupo conformado por: Uruguay, Costa Rica, Chile, Argentina, República Dominicana y México (6 países) presentan cifras mayores al 95\%. Entre ellos, Chile, Uruguay y Costa Rica están cercanos a una cobertura del $100 \%$ poblacional en ambos servicios.

La última comparación se realizó entre los años 2015 y 2017 (figuras 4 y 5). En este caso, Honduras se suma a los países con valores superiores al 90\% (10 países de 16); y Colombia al grupo por encima del 95\% poblacional para los 2 servicios.

Se reitera, que a nivel regional se aprecia una mejora en los dos indicadores en el trascurso de los 17 años. Para tener un análisis de esas mejoras, se hace lectura de la tasa anual de crecimiento.

Figura 1

Cobertura poblacional de fuentes mejoradas de agua y servicio sanitario seguro.

Países seleccionados de América Latina y el Caribe. Año 2000.

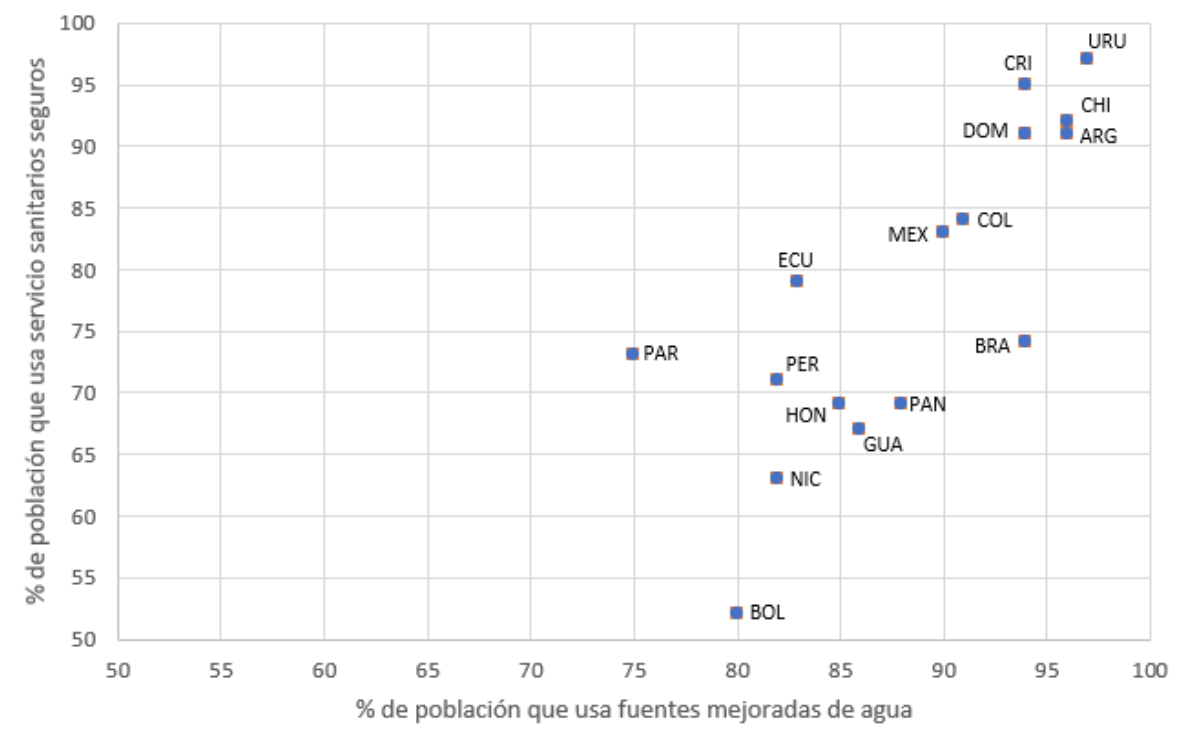

Fuente: autores, a partir de sdg6data.org 
Figura 2

Cobertura poblacional de fuentes mejoradas de agua y servicio sanitario seguro. Países seleccionados de América Latina y el Caribe. Año 2005.

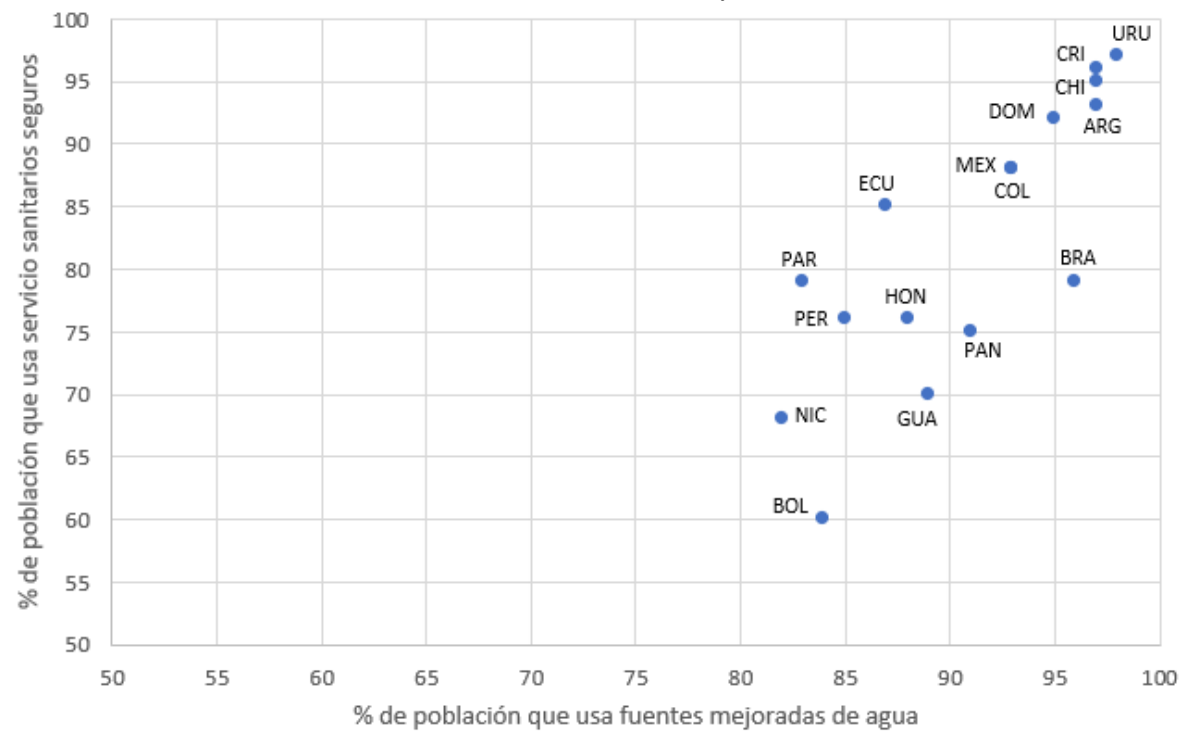

Fuente: autores, a partir de sdg6data.org

Figura 3

Cobertura poblacional de fuentes mejoradas de agua y servicio sanitario seguro. Países seleccionados de América Latina y el Caribe. Año 2010.

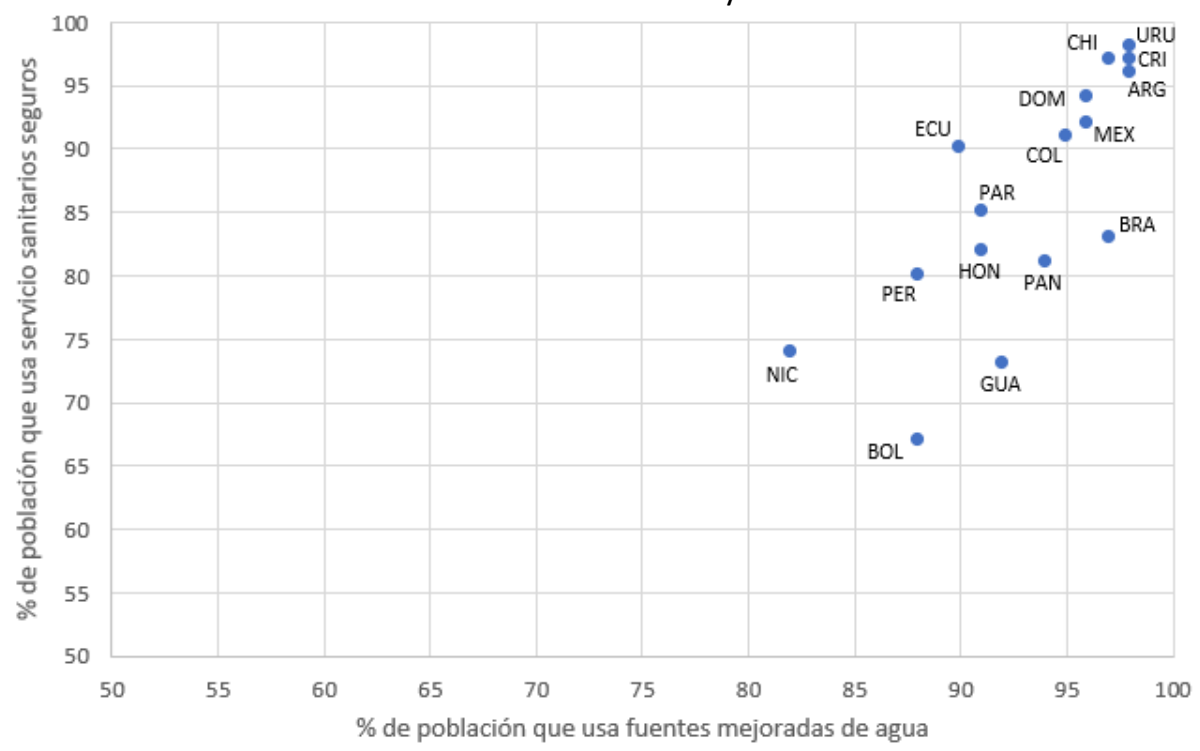

Fuente: autores, a partir de sdg6data.org 
Figura 4

Cobertura poblacional de fuentes mejoradas de agua y servicio sanitario seguro.

Países seleccionados de América Latina y el Caribe. Año 2015.

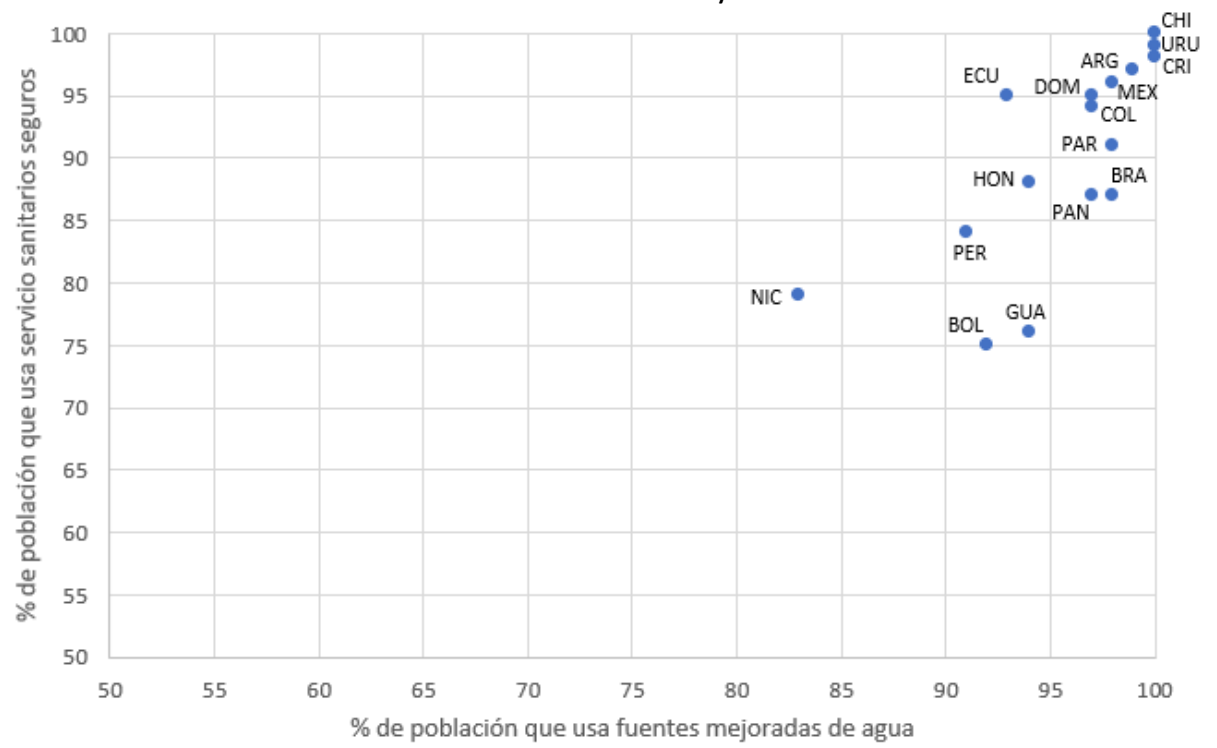

Fuente: autores, a partir de sdg6data.org

Figura 5

Cobertura poblacional de fuentes mejoradas de agua y servicio sanitario seguro.

Países seleccionados de América Latina y el Caribe. Año 2017.

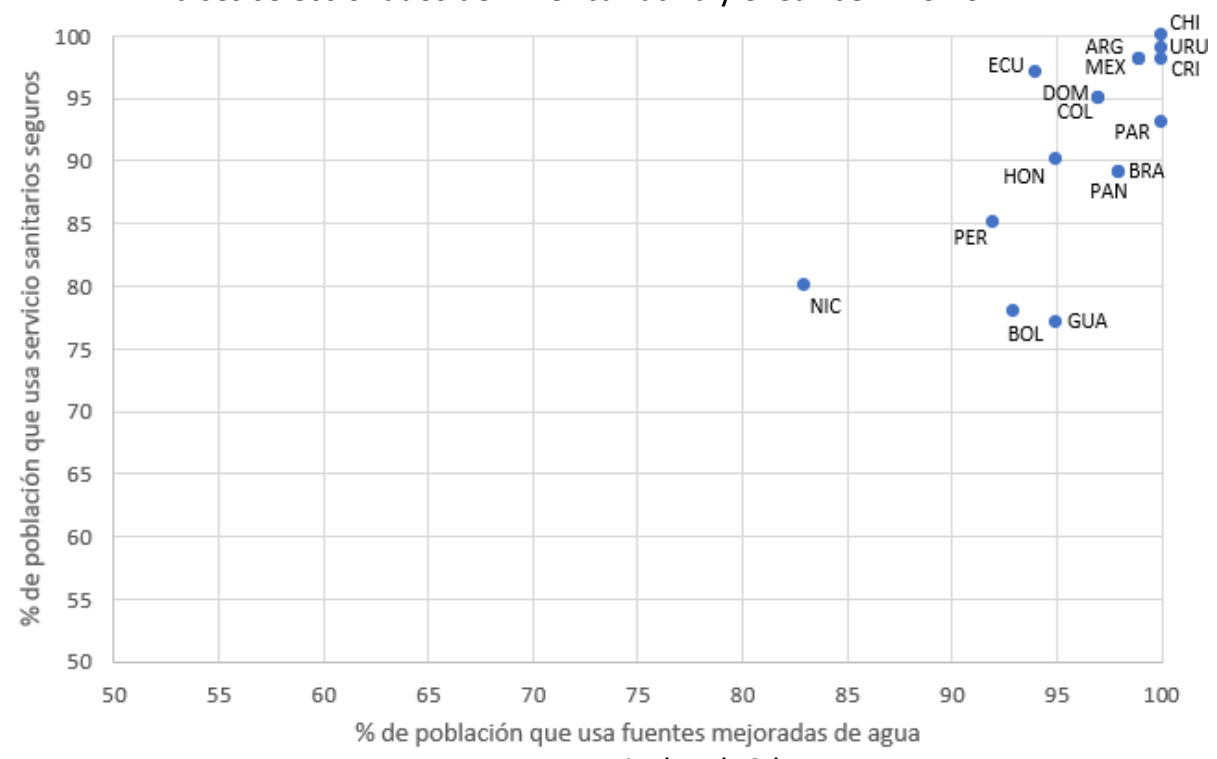

Fuente: autores, a partir de sdg6data.org

\subsection{Proyección de las tasas anuales de crecimiento. Ventana al año 2030.}

Inicialmente, para el indicador del \% de población que usa fuentes mejoradas de agua, se obtuvieron las siguientes tasas anuales de crecimiento de 2000 a 2017 (cuadro 1), para cada país: 
Cuadro 1

Tasa anual de crecimiento del \% de población que usa fuentes mejoradas de agua (2000 a 2017).

\begin{tabular}{|l|l|}
\hline Colombia & $0,39 \%$ \\
\hline Panamá & $0,67 \%$ \\
\hline Argentina & $0,18 \%$ \\
\hline Chile & $0,25 \%$ \\
\hline Brasil & $0,25 \%$ \\
\hline Perú & $0,72 \%$ \\
\hline Ecuador & $0,78 \%$ \\
\hline Bolivia & $0,96 \%$ \\
\hline Paraguay & $1,96 \%$ \\
\hline Uruguay & $0,18 \%$ \\
\hline México & $0,59 \%$ \\
\hline Costa Rica & $0,38 \%$ \\
\hline Nicaragua & $0,07 \%$ \\
\hline Honduras & $0,69 \%$ \\
\hline Guatemala & $0,62 \%$ \\
\hline Rep. Dominicana & $0,19 \%$ \\
\hline
\end{tabular}

Fuente: autores.

El avance de los indicadores del porcentaje de población que usa fuentes mejoradas de agua, y el porcentaje de población que usa servicio sanitario seguro; permiten establecer tasas de crecimiento anual. Cuadro 2 y cuadro 4, respectivamente.

Las proyecciones permiten establecer que para el indicador: el \% de población que usa fuentes mejoradas de agua, en el año 2017 solo Chile, Paraguay, Uruguay y Costa Rica habían logrado el 100\% de cobertura. Para el año 2020 (cuadro 2) se habrán sumado Argentina, Panamá y México. En el año 2025 Brasil, Bolivia, Colombia, Ecuador, Guatemala y Honduras habrán logrado esa misma meta. Finalmente, en 2030 será Perú el país que llegue al máximo porcentaje de cobertura. Pero para ese mismo año Nicaragua solo habrá llegado al 84\%, y República Dominicana llegará al 99\%. Esta última cifra ha de considerarse también como un logro del 100\%.

Cuadro 2

Valores proyectados del \% de población que usa fuentes mejoradas de agua

\begin{tabular}{|c|c|c|c|}
\hline $\begin{array}{c}\text { \% de población que usa fuentes } \\
\text { mejoradas de agua }\end{array}$ & $\begin{array}{c}\text { Proyectado a } \\
\mathbf{2 0 2 0}\end{array}$ & $\begin{array}{c}\text { Proyectado a } \\
\mathbf{2 0 2 5}\end{array}$ & $\begin{array}{c}\text { Proyectado a } \\
\mathbf{2 0 3 0}\end{array}$ \\
\hline Colombia & 98 & 100 & 102 \\
\hline Panamá & 101 & 104 & 107 \\
\hline Argentina & 100 & 101 & 101 \\
\hline Chile & 101 & 102 & 103 \\
\hline Brasil & 99 & 100 & 101 \\
\hline Perú & 95 & 98 & 102 \\
\hline Ecuador & 97 & 101 & 105 \\
\hline Bolivia & 97 & 101 & 106 \\
\hline Paraguay & 111 & 122 & 134 \\
\hline Uruguay & 101 & 102 & 102 \\
\hline
\end{tabular}




\begin{tabular}{|c|c|c|c|}
\hline $\begin{array}{c}\text { \% de población que usa fuentes } \\
\text { mejoradas de agua }\end{array}$ & $\begin{array}{c}\text { Proyectado a } \\
\mathbf{2 0 2 0}\end{array}$ & $\begin{array}{c}\text { Proyectado a } \\
\mathbf{2 0 2 5}\end{array}$ & $\begin{array}{c}\text { Proyectado a } \\
\mathbf{2 0 3 0}\end{array}$ \\
\hline México & 101 & 104 & 107 \\
\hline Costa Rica & 101 & 103 & 105 \\
\hline Nicaragua & 83 & 83 & 84 \\
\hline Honduras & 98 & 101 & 105 \\
\hline Guatemala & 97 & 100 & 103 \\
\hline Rep. Dominicana & 98 & 99 & 99 \\
\hline
\end{tabular}

Fuente: autores

En el cuadro 3 se indican las tasas anuales de crecimiento y posteriormente se realiza una lectura paralela para el indicador del \% de población que usa servicio sanitario seguro (cuadro 4).

Cuadro 3

Tasa anual de crecimiento del \% de población que usa servicio sanitario seguro (2000 a 2017).

\begin{tabular}{|l|l|}
\hline Colombia & $0,77 \%$ \\
\hline Panamá & $1,71 \%$ \\
\hline Argentina & $0,45 \%$ \\
\hline Chile & $0,51 \%$ \\
\hline Brasil & $1,19 \%$ \\
\hline Perú & $1,16 \%$ \\
\hline Ecuador & $1,34 \%$ \\
\hline Bolivia & $2,94 \%$ \\
\hline Paraguay & $1,61 \%$ \\
\hline Uruguay & $0,12 \%$ \\
\hline México & $1,06 \%$ \\
\hline Costa Rica & $0,19 \%$ \\
\hline Nicaragua & $1,59 \%$ \\
\hline Honduras & $1,79 \%$ \\
\hline Guatemala & $0,88 \%$ \\
\hline Rep. Dominicana & $0,26 \%$ \\
\hline
\end{tabular}

Fuente: autores

De manera similar, se calcularon las proyecciones permiten establecer que para el indicador: el \% de población que usa servicio sanitario seguro (cuadro 4). En el año 2017 solo Chile mostraba cobertura del 100\%. Luego, los registros del año 2020 agregan a Argentina, Ecuador, México y Paraguay. En el año 2025 Brasil, Bolivia, Colombia, Costa Rica, Honduras, Panamá y Uruguay habrán logrado esa misma meta. Finalmente, en 2030 serán Perú y Nicaragua los países que suban el indicador a $100 \%$. No obstante, el esfuerzo de todos los países por mejorar el indicador, Guatemala y República Dominicana llegan al $87 \%$ y $98 \%$, respectivamente. Claro está, sin ceñirse a la rigurosidad de las cifras, se otorga a República Dominicana el logro de una cobertura total. 
Cuadro 4

Valores proyectados del \% de población que usa servicio sanitario seguro

\begin{tabular}{|c|c|c|c|}
\hline $\begin{array}{c}\text { \% de población que usa } \\
\text { servicios sanitarios seguros }\end{array}$ & $\begin{array}{c}\text { Proyectado } \\
\text { a 2020 }\end{array}$ & $\begin{array}{c}\text { Proyectado } \\
\text { a 2025 }\end{array}$ & $\begin{array}{c}\text { Proyectado } \\
\text { a 2030 }\end{array}$ \\
\hline Colombia & 98 & 102 & 106 \\
\hline Panamá & 97 & 105 & 115 \\
\hline Argentina & 100 & 102 & 104 \\
\hline Chile & 102 & 105 & 107 \\
\hline Brasil & 94 & 100 & 106 \\
\hline Perú & 89 & 95 & 100 \\
\hline Ecuador & 103 & 110 & 118 \\
\hline Bolivia & 93 & 107 & 124 \\
\hline Paraguay & 101 & 109 & 118 \\
\hline Uruguay & 99 & 100 & 101 \\
\hline México & 103 & 108 & 114 \\
\hline Costa Rica & 99 & 100 & 100 \\
\hline Nicaragua & 86 & 93 & 101 \\
\hline Honduras & 98 & 108 & 118 \\
\hline Guatemala & 80 & 83 & 87 \\
\hline Rep. Dominicana & 96 & 97 & 98 \\
\hline
\end{tabular}

Fuente: autores.

\subsection{Diferenciación de los países analizados}

Si se identifican los países que lograrían obtener en 2017 cobertura poblacional en ambos servicios por encima del 95\%: Chile, Uruguay, Costa Rica, México, Colombia, Argentina y República Dominicana; solo Chile y Uruguay están en el grupo de países con PIB per cápita mayor a U\$20.000. Los otros países mencionados estarían en un segundo grupo (de 3 grupos, con límites cada U\$ 10.000) de mayor PIB per cápita entre los U\$ 10.000 y U\$ 20.000 .

Ahora, si se compara ese mismo grupo con los registros de porcentaje de población urbana en 2017, todos están con registro superior al $75 \%$.

Continuando el análisis por grupos, los países que logran para el año 2017 una cobertura conjunta de ambos servicios entre el $90 \%$ y $95 \%$ son: Ecuador, Honduras y Paraguay. Ecuador y Paraguay están en el grupo de países con PIB per cápita superior a U\$ 10.000, pero menor a U\$20.000; y Honduras presenta el PIB más bajo en la muestra de países. Nuevamente, no hay una relación estadística significativa entre: la situación económica de un país latinoamericano y del Caribe, y la condición de los 2 servicios públicos.

Pero, si se compara este segundo grupo de países con su porcentaje de población urbana, los 3 se encuentran en el grupo con los porcentajes más bajos: por debajo del $65 \%$.

Finalmente, si se segrega un tercer grupo de países como aquellos que no lograron en 2017 tener cobertura superior al $90 \%$ en ambos servicios públicos, se tiene: Bolivia, Brasil, Panamá, Perú, Guatemala y Nicaragua. Al comparar con el PIB per cápita, se tiene que Panamá tiene el segundo PIB más alto de la muestra (mayor a U\$ 20.000), Brasil y Perú están en un segundo grupo con PIB entre U\$ 10.000 y U\$20.000, mientras que Bolivia, Guatemala y Nicaragua están entre los menores PIB de la muestra (menor a U\$ 10.000). En el análisis por porcentaje de población urbana, Brasil es el único que presenta un registro superior al $80 \%$, Perú está levemente 
por debajo de ese porcentaje, pero los cuatro países registran ese indicador por debajo del 70\%, quedando en el grupo con valores más bajos.

Por tanto, se concluye de este apartado que no hay una clara relación estadística que permita establecer que los países que han logrado mayores coberturas poblacionales para los 2 servicios corresponden a los países con mayores recursos económicos y mayor facilidad por tener una importante concentración de población urbana. Una mayor tasa de población urbana podría inferir que el esfuerzo en extensión de redes de servicios es menor que teniendo que llevarla a zonas rurales; y por ende, se tendría una mejor cobertura. El análisis de la condición económica del país (PIB per cápita) llevaría a inferir también una mayor cobertura de servicios.

\subsection{Correlación entre economía, población urbana, y mejora en la cobertura de servicios}

Una inquietud que surge tras el cálculo de las tasas anuales de cobertura poblacional de los servicios, es si existe una correlación de variables que identifique a los países que tuvieron mayores tasas promedio de avance entre 2000 y 2017 , y a aquellos que menos.

Para ello se establecieron 2 indicadores adicionales: la tasa media anual del crecimiento del PIB per cápita (a valores constantes) y la tasa media anual del crecimiento de población urbana. Estas cifras ayudan a explicar las condiciones con que contaron los países que mayor cobertura de servicios lograron en la ventana de tiempo estudiada. Las cifras quinquenales para ambos indicadores, calculados entre los años 2000 y 2019 , se obtuvieron de la base de datos y publicaciones estadísticas de la CEPAL $(2020$, b). Paso a seguir se calcularon las tasas de crecimiento media anual para cada país (Cuadro 5).

\section{Cuadro 5}

Tasa media anual porcentual de población urbana, y de PIB per cápita (2000-2019)

\begin{tabular}{|c|c|c|}
\hline & $\begin{array}{c}\text { Tasa media anual } \\
\text { Pob. Urb. - 00-19 }\end{array}$ & $\begin{array}{c}\text { Tasa media anual } \\
\text { PIB per cápita - 00-19 }\end{array}$ \\
\hline Colombia & $0,5 \%$ & $3 \%$ \\
\hline Panamá & $0,5 \%$ & $4 \%$ \\
\hline Argentina & $0,2 \%$ & $1 \%$ \\
\hline Chile & $0,2 \%$ & $3 \%$ \\
\hline Brasil & $0,4 \%$ & $1 \%$ \\
\hline Perú & $0,5 \%$ & $4 \%$ \\
\hline Ecuador & $0,5 \%$ & $2 \%$ \\
\hline Bolivia & $0,8 \%$ & $3 \%$ \\
\hline Paraguay & $1,1 \%$ & $2 \%$ \\
\hline Uruguay & $0,3 \%$ & $3 \%$ \\
\hline México & $0,2 \%$ & $1 \%$ \\
\hline Costa Rica & $1,9 \%$ & $3 \%$ \\
\hline Nicaragua & $0,3 \%$ & $2 \%$ \\
\hline Honduras & $1,3 \%$ & $2 \%$ \\
\hline Guatemala & $2,0 \%$ & $1 \%$ \\
\hline Rep. Dom. & $1,8 \%$ & $4 \%$ \\
\hline
\end{tabular}

Fuente: autores

El primer análisis que se realizó fue correlacionando indicadores recientes: \% de población que usa fuentes mejoradas de agua en 2017, PIB per cápita 2018 (precios constantes base U\$ 2010) y \% población urbana 2017; como variables explicativas de la tasa promedio anual de la población que usa fuentes mejoradas de agua, entre el año 2000 y 2017. El resultado no fue satisfactorio, pues se obtuvo un $R^{2}$ ajustado de 0.40 . Luego se combinaron 
las variables explicativas de 2 en 2 y de manera individual, pero nuevamente los $R^{2}$ ajustado en las regresiones oscilaron en valores cercanos a 0.45 , en el mejor de los casos.

De manera paralela se realizó la correlación para las mismas variables explicativas, pero respecto de la tasa media anual de \% de población que usa servicios sanitarios seguros entre 2000 y 2017 . El valor $\mathrm{R}^{2}$ ajustado de las distintas regresiones multilineales no superó 0.35 . Por tanto, tampoco se presentó una correlación estadísticamente considerable.

Con los resultados obtenidos, no puede afirmarse que los países que tuvieron mayores avances en la cobertura poblacional de los servicios públicos (en estudio), son aquellos que lograron obtener mejor economía, o que tuviesen mayor proporción de población urbana, o que lograron mejores cifras de cobertura a 2017. Es decir, los países que realizaron "mayores esfuerzos" y lograron mejores tasas en el crecimiento de su cobertura, no son los países con mejor PIB de la región. Pero hay que tener cuidado porque tampoco puede afirmarse lo contrario, o simplemente se hubiese obtenido un $\mathrm{R}^{2}$ ajustado satisfactorio (mayor a 0.75 ) con coeficientes de correlación de signos contrarios a los obtenidos en este planteamiento.

Lo anterior llevó a realizar otro planteamiento: que los países que registraron mayores tasas anuales de cobertura en servicios, entre los años 2000 y 2017, fueron aquellos que tenían los más altos registros de cobertura en el año 2000; es decir, que estaban más cerca de cumplir con los ODM en materia de agua y saneamiento. Los resultados en materia de fuentes mejoradas de agua, fue de $\mathrm{R}^{2}$ ajustado de 0.68 para las variables explicativas: \% de población que usa fuentes mejoradas de agua en el año 2000 y el PIB per cápita en 2018. Si bien hay mayor relación explicativa comparado con el planteamiento de los párrafos anteriores, no tiene un valor que estadísticamente de confianza.

Nuevamente, se realizó el paralelo para el tema saneamiento. En esta ocasión el $R^{2}$ ajustado fue de 0.80 . Con este valor de correlación puede afirmarse que en el caso saneamiento, los países de la muestra regional que tuvieron mejores tasas o avances en cobertura poblacional de los 2 servicios públicos, fueron aquellos que tenían las mejores coberturas en el año 2000 y que tenían mejor potencial económico (medido a partir del PIB per cápita) y contaban con mayor proporción de población urbana.

Pero el análisis anterior tenía aún un cambio por realizar. Si bien la correlación fue válida estadísticamente, los registros de PIB y población urbana eran para el final del período (2017 y 2018). Ahora, faltaba estudiar si la correlación es mejor si se analizan los países como aquellos que tuvieron mejores tasas de crecimiento para esas dos variables, a partir del año 2000.

Por tanto, se procedió a correlacionar si los países con mejor tasa media anual del porcentaje de población que usa fuentes mejoradas de agua son aquellos que tuvieron mejor comportamiento o tasa media anual de crecimiento económico y de población urbana (una visión dinámica en 19 años, y no solo la lectura de fin del período). Los resultados al combinar las variables explicativas no superaron un $\mathrm{R}^{2}$ ajustado de 0.54 . De nuevo, la cobertura en el servicio de fuentes de agua no encuentra explicación regional con la facilidad de tener más población en ciudades y mejor condición económica.

Luego se realizó el análisis paralelo con la tasa media anual de la población que usa servicios sanitarios seguros. En esta ocasión la correlación resultó favorable, pues el $\mathrm{R}^{2}$ ajustado fue de 0.82 , al explicarse con el porcentaje de cobertura del servicio en el año 2000, el avance anual en la condición económica (PIB per cápita) y el avance anual en la proporción de población urbana.

La ecuación que explica el modelo de regresión anterior es:

$$
Y=0.05-\left(0.0005^{*} \times 1\right)+(0.020 * x 2)-\left(0.18^{*} \times 3\right) \quad \text { Ec. } 2
$$


Donde:

$Y=$ Tasa promedio anual del porcentaje de población que accede a servicios sanitarios seguros

X1 = Porcentaje de población que usa servicios sanitarios seguros (2000)

X2 = Tasa promedio anual del incremento en PIB per cápita (2000-2019)

X3 = Tasa media anual de la proporción de población urbana (2000-2019)

Cabe anotar que los coeficientes para $x 1$ y x3 dieron negativos, lo cual se interpreta como que los países que más incrementaron en tasa media anual el porcentaje de población que accede a servicios sanitarios seguros, son los países que tuvieron menos:

a. Porcentaje de población que usa servicios sanitarios seguros (2000), y

b. Tasa media anual de la proporción de población urbana (2000-2019)

Lo primero implica un menor porcentaje de población que demanda cobertura, y lo segundo, que había una alta cantidad de población en ciudades en el año 2000, y el crecimiento de ese indicador fue bajo en los 19 años. El signo positivo de $\times 2$ se traduce en que esos países que lograron mejor tasa de cobertura tenían buen comportamiento económico.

Revisando cifras, los países que registraron mejores tasas de cobertura poblacional entre los años 2000 y 2019 son: Bolivia, Honduras, Panamá, Paraguay, Nicaragua y Ecuador. Todos tuvieron crecimiento medios anuales del PIB per cápita mayores al $2 \%$. Con excepción de Honduras y Paraguay, el crecimiento medio anual del porcentaje de población urbana está por debajo del 1\%; y la cobertura del servicio en el año 2000 estaba por debajo del $80 \%$ de la población.

Por lo contrario, los países que tuvieron incrementos más bajos de la tasa de cobertura poblacional en servicios sanitarios seguros fueron: Uruguay, Costa Rica, República Dominicana, Argentina y Chile; con registros por debajo de $0.5 \%$. Esos son los países de la muestra que tenían una cobertura del servicio en el año 2000 superior al $90 \%$. También cabe anotar que, con excepción de Argentina, este grupo tuvo altas tasas regionales en el crecimiento del PIB per cápita (3\% y 4\%); y con excepción de Costa Rica y República Dominicana, las tasas de crecimiento anual de población urbana registran por debajo de $0.3 \%$.

Finalmente, se correlacionaron las mismas variables, pero tomando al incremento anual porcentual del PIB per cápita como la variable que se explica por el avance en la tasa anual de población urbana y de la cobertura del servicio de saneamiento. En este caso los resultados mostraron que no hay correlación alguna, pues los valores de $\mathrm{R}^{2}$ ajustado dieron negativos.

Las correlaciones realizadas ponen de manifiesto que el avance en la cobertura poblacional de servicios de saneamiento, no coadyuvan al mejoramiento económico de un país (reflejado en su PIB), pero que un país de la región con una economía en buenas condiciones, y con una cobertura poblacional avanzada en los servicios logra mejorar significativamente el saldo poblacional pendiente por abastecer.

El hecho de que la correlación no sea del mismo nivel en el servicio de abastecimiento de agua se debe a que la red de agua, desde el año 2000, se encontraba en mayor grado de cobertura que la de saneamiento, y por tanto, el esfuerzo de los países de la muestra, se ha enfocado más en el segundo servicio. 


\section{Conclusiones}

Como se conoce desde estudios referentes a períodos de tiempo anteriores en el avance de los Objetivos del Milenio (ODM), la cobertura poblacional de los servicios de agua es superior a la de los servicios asociados a saneamiento básico.

En una lectura evolutiva por quinquenios entre los años 2000 y 2015, y luego entre 2015 y 2017, se aprecia la mejora en cobertura poblacional de los servicios de agua y saneamiento en condiciones seguras. El grupo de países seleccionados permite identificar a nivel gráfico a aquellos que tuvieron niveles de cobertura que se aproximaban al 90\% y 95\%, y por tanto, lograron aproximar sus 2 indicadores al 100\%, o se prevé (por proyección del comportamiento histórico) que lo lograrán en los próximos años. Con la misma facilidad se denota un segundo grupo de países cuya cobertura para ambos servicios oscila entre el $80 \%$ y $90 \%$. Finalmente, un tercer grupo de países tiene la importante tarea de obtener registros importantes, cuando a principios de la década del 2000 , tenían valores inferiores al $80 \%$.

La proyección de las tendencias históricas de cobertura poblacional, a partir del año 2000, permite establecer que para el año 2030 (límite para el cumplimiento de los ODS), (1 solo país de 16 que conforman la muestra) no habrá logrado el cometido de una cobertura de los servicios al $100 \%$ de la población, o se habrá aproximado a ello; tanto en el caso de agua como en el de saneamiento.

Ahora, en la relación de los porcentajes de cobertura poblacional de los servicios en estudio, con el nivel económico (PIB per cápita) y la necesidad de invertir más en zonas urbanas que rurales (\% de población urbana) no se encontró una relación que indicara que los países con mayores recursos económicos y mayor concentración poblacional urbana, tuvieran los mejores registros de cobertura en los servicios.

El análisis comparativo entre cobertura de servicios, economía, y población urbana se complementó con una lectura evolutiva de las tasas medias anuales de crecimiento para las 3 variables. El resultado obtenido es que la cobertura en el servicio de fuentes de agua no encuentra explicación regional con la facilidad de tener más población en ciudades y mejor condición económica. Pero, para el servicio de saneamiento mejorado la correlación multilineal resultó en un $\mathrm{R}^{2}$ ajustado de 0.82 . Ahora sí hay relación entre los avances en los 3 indicadores. Los países que lograron mejor tasa de cobertura tuvieron buen comportamiento económico desde la década del año 2000.

Finalmente, se planteó la correlación en la cual los mejores comportamientos en avance de cobertura de los servicios tendrían impacto en el comportamiento del PIB per cápita. El resultado es que no hay relación que se valide estadísticamente para este caso. Es decir, el que un país de la región cuente con amplia cobertura de servicios a su población, no implica que haya una retribución económica; pero un país con buen nivel económico si tiene instrumentos para avanzar en la extensión de sus redes de servicios básicos. Y esa relación está marcada hacia la cobertura del servicio de saneamiento, pero no tanto para el caso del servicio de abastecimiento de agua.

Pero la relevancia de que aún haya una cantidad importante de población mundial sin acceso a los servicios estudiados, son las externalidades negativas referentes a salubridad y calidad de vida, pues generalmente la carencia de estos servicios viene ligado a problemas de vivienda (a nivel edificación) y hábitat (deficiencia en espacio público y accesibilidad al asentamiento humano). Esto relaciona los avances en el ODS 6 con los avances parciales en el ODS 11. 


\section{Referencias bibliográficas}

Banco de Desarrollo de América Latina CAF (2012). Agua potable y saneamiento en América Latina y el Caribe: metas realistas y soluciones sostenibles. Caracas, $56 \mathrm{p}$.

Banco Interamericano de Desarrollo BID (2020). Cómo está América Latina en términos de saneamiento? https://www.iadb.org/es/mejorandovidas/como-esta-america-latina-en-terminos-de-saneamiento. Revisado en octubre de 2020.

Castro, F. y Valbuena, N. (2020). Índice 2019 para América Latina y el Caribe. Centro de los Objetivos de Desarrollo Sostenible para América Latina y el Caribe. Bogotá, 135 p.

Consejo Nacional de Política Económica y Social CONPES (2018). Documento CONPES 3918: Estrategia para la implementación de los Objetivos de Desarrollo Sostenible ODS en Colombia. Bogotá, 74 p.

Comisión Económica para América Latina y el Caribe CEPAL, Organización de las Naciones Unidas ONU (2017). Desarrollo sostenible, urbanización y desigualdad en América Latina y el Caribe. Dinámicas y desafíos para el cambio estructural. Santiago, 427 p.

Comisión Económica para América Latina y el Caribe CEPAL, Organización de las Naciones Unidas ONU (2020 a). Estado del avance de los indicadores del ODS 6: situación de América Latina y el Caribe. Recuperado de: https://www.cepal.org/sites/default/files/events/files/ods_6_en_alc_cepal_-_undesa_cr_mg.pdf

Comisión Económica para América Latina y el Caribe CEPAL, Organización de las Naciones Unidas ONU (2020 b). Base de datos y publicaciones estadísticas CEPALSTAT. Recuperado de: https://estadisticas.cepal.org/cepalstat/web_cepalstat/estadisticasIndicadores.asp?string_busqueda=pib

Organización Mundial de la Salud OMS (2017). Radical increase in wáter and sanitation investment required to meet development targets. Recuperado de: https://www.who.int/news/item/13-04-2017-radicalincrease-in-water-and-sanitation-investment-required-to-meet-development-targets

Organización de las Naciones Unidas ONU (2015). Objetivos del Desarrollo del Milenio. Informe de 2015. Nueva York, $75 \mathrm{p}$.

Organización de las Naciones Unidas ONU (2020 a). Recuperado de: https://www.un.org/sustainabledevelopment/es/objetivos-de-desarrollo-sostenible/

Organización de las Naciones Unidas ONU (2020 b). Sustainable Development Goals Fund. Recuperado de: https://www.sdgfund.org/es/de-los-odm-los-ods

Organización de las Naciones Unidas ONU (2020 c). Recuperado de: https://www.un.org/sustainabledevelopment/es/progress-report/. Revisado en agosto 2020.

Organización de las Naciones Unidas ONU (2020 d). Recuperado de: https://www.un.org/sustainabledevelopment/es/water-and-sanitation/. Revisado en agosto 2020.

Organización de las Naciones Unidas ONU (2020 e). Agua limpia y saneamiento: porqué es importante? Recuperado de: https://www.un.org/sustainabledevelopment/es/wpcontent/uploads/sites/3/2016/10/6_Spanish_Why_it_Matters.pdf

Paltán, H.; Bazani, M; Minaya, V. y Rezzano, N. (2020). Servicios de agua potable y saneamiento resilientes en América Latina y El Caribe. Banco Interamericano de Desarrollo BID. 57 p. Recuperado de: 
https://publications.iadb.org/publications/spanish/document/Servicios-de-agua-potable-y-saneamientoresilientes-en-America-Latina-y-el-Caribe.pdf.

Pinilla, D. y Torres, Y. (2019). Social Public Social Expenditure, Access to Drinking Water and Sanitation for Rural Populations in Latin America. Revista Problemas del desarrollo, Vol. 50, No. 196. México D.F., 28 p.

Programa de las Naciones Unidas para el Desarrollo PNUD (2020 a). Recuperado de: https://www.undp.org/content/undp/es/home/sdgoverview/mdg_goals.html.

Programa de las Naciones Unidas para el Desarrollo PNUD (2020 b). Recuperado de: https://www.undp.org/content/undp/es/home/sustainable-development-goals/goal-11-sustainablecities-and-communities.html.

Swiss International Water Institute SIWI (2019). Latin America and the Caribbean: Optimal sanitation for all. https://www.worldwaterweek.org/press/resources. Revisado en: octubre de 2020.

Tezanos, S. (2019). América Latina y el Caribe en la agenda 2030. Hacia una clasificación del desarrollo sostenible compatible con los ODS y el "desarrollo en transición”. Fundación Carolina. Madrid, 24 p.

Urazán, C.F. (2008). El rol de los servicios públicos básicos en las formas de crecimiento urbano. Estudio de caso de la ciudad de Cúcuta, Colombia. Universidad Politécnica de Cataluña, Tesis doctoral. Barcelona, p.409.

Urazán, C.F. y Caicedo, M.A. (2018). Modelo de correlación entre economía, población y servicios de agua y saneamiento en América Latina. Análisis de 1990 a 2015. Revista Espacios, Vol. 39, No. 9, p. 19. ISSN 07981015.

Urazán, C.F.; Caicedo, M.A.; Correa, F.M.; Valcarcel, P.A. (2018). Análisis comparado del aumento de población urbana con la mejora en la cobertura de servicios públicos domiciliarios de agua y saneamiento, caso Latinoamérica. Academia e investigación en perspectiva interdisciplinar. Universidad de La Salle, Bogotá, pp. 58-71.

Esta obra está bajo una Licencia Creative Commons Attribución-NoCommercial 4.0 International

(cc) BY-NC 